\title{
Taranenko G.
}

\section{ANALYSIS OF DUAL-FLOW PLATES WITH A LARGE FREE SECTION}

The object of research is the dual-type plates with a large free section. One of the most problematic areas of such plates is their low efficiency, which depends on the operating mode of the dual-type plates with a large free section. The gas-liquid layer on such plates is represented by drops and films of liquid, which are the dispersed phase. In this case, the continuous phase is gas. Insufficient liquid supply on dual-flow plates with a large free section leads to a decrease in the efficiency of such plates.

During the research, the method of hydrodynamic modeling was used. This method consists in the fact that the dual-flow plates are investigated first in experimental installations, and their results, according to a certain method, are used to calculate industrial plates.

A study of dual-flow plates with a large free section in a wide range of gas and liquid loads has been carried out. The range of work of the plates has been determined. It has been established that the hydraulic resistance of the dual-flow plates with a large free section depends little on the diameter of the column apparatus. An almost complete coincidence of the dependence of the height of the gas-liquid layer on the gas velocity in the column cross-section is shown for dual-flow plates with a large free section installed in columns with diameters $D=0.057,0.4$ and $2 \mathrm{~m}$. It is revealed that a column with a diameter of $D=0.057 \mathrm{~m}$ can be used to simulate the operation of dual-flow plates with a large free section of industrial diameter. For example, for dual-flow plates of the usual free section during hydrodynamic modeling, it is necessary to use pilot plants with columns of larger diameter $D=0.15-0.25 \mathrm{~m}$ with a large free cross-section can operate at significantly higher gas and liquid velocities compared to the dual-flow plates with a conventional free cross-section.

The use of dual-flow plates with a large free section is recommended in absorption processes at high irrigation densities.

Keywords: dual-flow plate, large free section of the column, hydraulic resistance, height of the gasliquid layer.

\section{Introduction}

Mass transfer trays are widely used in chemical, petrochemical, food and other industries. Most often they are used in the processes of rectification of various products.

The dual-flow plates have a minimum manufacturing cost, are convenient for installation and operation, the ability to work with contaminated media.

The dual-flow plates with a large free section allow high linear velocities of both gas and liquid. This allows to design columns with a smaller diameter with the same performance.

The disadvantages of such plates include a relatively low efficiency of their work.

An urgent task is to study the efficiency of mass transfer on dual-flow plates with a large free section.

\section{The object of research and its technological audit}

The object of research is the dual-flow plates with a large free section. This section is $f>0.3$. The plates are made of different materials with holes of different diameters. In this work, plays with a hole diameter $d=0.012 \mathrm{~m}$ were studied.
Such plates allow high linear gas velocities $w=2-4 \mathrm{~m} / \mathrm{s}$ at different irrigation densities $L=100-5 \mathrm{~m}^{3} /\left(\mathrm{m}^{2} \cdot\right.$ hour $)$, respectively.

One of the most problematic areas of such plates is the relatively low efficiency of their work, as well as work with a large amount of spray loss.

\section{The aim and objectives of research}

The aim of research is to investigate a dual-flow plate with a large free section.

To achieve the aim, the following objectives have been set:

1. Set the operating range of the dual-flow plates with a large free section.

2. Establish the dependence of the hydraulic resistance of the dual-flow plates with a large free section on the gas velocity in the column section and the density of reflux in liquid.

3. Determine the dependence of the height of the gasliquid layer on dual-flow plates with a large free section on the gas velocity in the column section and the density of reflux in liquid.

4. Establish the area of application of dual-flow plates with a large free section. 


\section{Research of existing solutions of the problem}

Several models for calculating the efficiency of mass transfer on mass transfer plates are considered in the literature.

In [1], to calculate the efficiency point, a model of the foam structure was proposed based on the analysis of the image of the foam taken on a dual-flow sieve tray with a diameter of $0.153 \mathrm{~m}$ of the distillation column. Foam has been defined as a combination of bubbles and continuous jets that break the surface of the foam, resulting in splashes of liquid falling onto the foam surface. It is noted that the simultaneous transfer of mass and heat, combined with a complex two-phase liquid movement, makes distillation difficult for fundamental analysis.

The efficiency of mass transfer on a dual-flow type is related to liquid dynamics. This determines the structure of the dispersion or the area of contact between the gas and liquid phases. Modes of movement on a dual-flow plate directly affect the efficiency by acting on the interface. The conventionally perceived pattern is that the foam regime consists of bubbles in the liquid, which is a continuous phase, and the spray regime, in which the gas-liquid layer consists of droplets in the gas continuous phase. These modes for foam and spray determine the sudden change in the nature of the two-phase mixture in the transition zone and involve two separate expressions to predict the effectiveness of the plate in these two modes.

The overall efficiency is estimated by adding the contributions of both bubbles and jets present in the dispersion. And the performance data for the dual-flow plate, on the other hand, shows a smooth transition of the plate efficiency from the hanging point to the flooding point of the plate. This led many researchers to come up with a uniform model of plate performance for foam and spray modes. Most of the existing models of plate efficiency [2-4] are based on a single efficiency model.

In [1], the foam mode is modeled based on the analysis of the image of the foam obtained on a plate with a diameter of $0.153 \mathrm{~m}$. The model describes the foam as a combination of bubbles and continuous jets. At higher gas load, the jet fraction dominates and transforms into spray mode. This foam model is additionally adopted to develop a baseline model that predicts the performance of the dual-flow plate. The efficiency of the model takes into account the contribution of the bubbling and jet operating modes to the overall mass transfer. On the basis of the adopted structure of the bubbling layer on a dual-flow plate, the method for assessing the total point efficiency $E_{O G}$ is shown in the form of equations (1) and (2). The total point efficiency can be determined by adding the efficiency of the bubble and jet zones that exist on the plate.

$$
E_{O G}=\left(1-f_{j}\right) E_{B}+f_{j} E_{j},
$$

where $f_{j}$ - the volume fraction of gas that bypasses the bubbles in the form of continuous jets; $E_{B}$ and $E_{j}$ are the contribution of the bubble and jet zones, respectively, to the overall point efficiency $E_{O G}$.

Due to the incomplete disintegration of large (primary) bubbles, both large (primary) and small (secondary) bubbles coexist in the bubbling zone. Thus, $E_{B}$ has the contribution of both large and small bubbles:

$$
E_{B}=(1-F S B) E_{L B}+F S B \cdot E_{S B}
$$

where $F S B$ - fraction of small bubbles; $E_{L B}$ - general point efficiency for large bubbles; $E_{S B}$ - total point efficiency for small bubbles.

In turn, point efficiencies $E_{B}$ and $E_{j}$ are calculated using equations derived from the general theory of distillation of binary mixtures.

In [3], a model for predicting the point efficiency for cross-type sieve trays was developed. It is indicated that vapor-liquid mixing models are required to convert Murfrey tray efficiency to point efficiency. The study of the geometry of the test plate led to the conclusion that the vapor is likely to be well mixed between the plates, since the distances between the plates and the diameter were relatively large. Departing from this assumption will not have much impact on the final model [5]. The effects of mixing a liquid can be taken into account by the vortex diffusion model [6].

The model provides for the calculation of the point efficiency through the Murfrey efficiency:

$$
\begin{aligned}
& \frac{E_{m v}}{E_{o v}}=\frac{1-e^{-\left(n^{\prime}+P e\right)}}{\left(n^{\prime}+P e\right)\left(1+\frac{n^{\prime}+P e}{n^{\prime}}\right)}+\frac{e^{n^{\prime}}-1}{n^{\prime}\left(1+\frac{n^{\prime}}{n^{\prime}+P e}\right)}, \\
& n^{\prime}=\frac{P e}{2}\left(\left[1+\frac{4 \lambda E_{o v}}{P e}\right]^{1 / 2}-1\right),
\end{aligned}
$$

where $E_{m v}-$ Murfrey efficiency, calculated from the concentration in the vapor phase; $E_{o v}$ - point efficiency, calculated from concentrations in the vapor phase; $P e$ - Peclet number; $\lambda$ - ratio of the slope of the balance line to the working line.

The Peclet number is determined by the equation:

$$
P e=\frac{Z_{l}^{2}}{D_{E} \bar{t}_{L}},
$$

where $Z_{l}$ - length of the liquid stroke, $\mathrm{m} ; D_{E}$ - coefficient of vortex diffusion, $\mathrm{m}^{2} / \mathrm{s} ; \bar{t}_{L}$ - average residence time of the liquid, $s$.

The path length $Z_{l}$ is taken as the distance between the inlet and outlet of the liquid on the plate.

For very low $P e$ values (high diffusion backmixing), equation (3) is reduced to an ideal mixing model where:

$$
\frac{E_{m v}}{E_{o v}}=1
$$

For very high $P e$ values, equation (3) is reduced to a plug flow model and the point efficiency in this case is calculated by the equation:

$$
E_{o v}=\frac{\ln \left(\lambda E_{m v}+1\right)}{\lambda}
$$

The vortex diffusion coefficient $D_{E}$ in the equation must be measured experimentally.

The average liquid residence time in equation (5) is based on the foam retention on the plate:

$$
\bar{t}_{L}=\frac{h_{L} A_{a}}{100 Q},
$$

where $h_{L}$ - liquid retention on the plate, cm; $A_{a}$ - active or bubbling area of the plate, $\mathrm{m}^{2} ; Q$ - liquid flow rate, $\mathrm{m}^{3} / \mathrm{s}$.

The value $h_{L}$ is determined according to the method developed in [7]. 
From works [1, 3], the following conclusions can be drawn: - models have been developed to predict the point efficiency of both the dual-type plates [1] and crosstype sieve trays [3];

- in [1] the model is created on the basis of the description of the structure of the gas-liquid layer on the dual-flow plate, and in [3] the diffusion model of the operation of the mass transfer plate is used;

- in [1,3] the models are created on the basis of data obtained in the process of rectification of various binary systems;

- in $[1,3]$, to create models of point efficiency, the work of plates of the usual free section $f<0.25$ is used.

In [8], it is noted that three characteristic hydrodynamic regimes are distinguished on the dual-flow plates:

1) gas is distributed in the liquid;

2) phase inversion;

3) liquid is distributed in the gas.

The boundaries of the indicated hydrodynamic regimes depend on the hydrodynamic, physicochemical, and design parameters of the system and can vary within wide limits.

Among the geometrical parameters, there are dual-flow plates with a small free section $f \leq 0.25$ and a large free section $f>0.3$. For dual-flow plates with a large free section, it is impossible to trace the change in hydrodynamic regimes with increasing gas velocity. Almost up to the flooding point, a very low gas-liquid layer with thin constantly bursting liquid films is formed on them. A characteristic feature of such plates, especially at high linear gas velocities $(w>1-2 \mathrm{~m} / \mathrm{s})$, is intense splashing, since there is practically no separating layer of gas-liquid foam [8].

In [9], the operation of dual-flow plates with a large free cross section is considered. Mass transfer on dual-flow plates is studied during the rectification of the methanol water (50 mol. \%) system.

Fig. 1 shows the graphs of the dependence of the efficiency of dual-flow plates with a large free section $(f=0.36$ and $f=0.56$ ) on the vapor velocity in the column section.

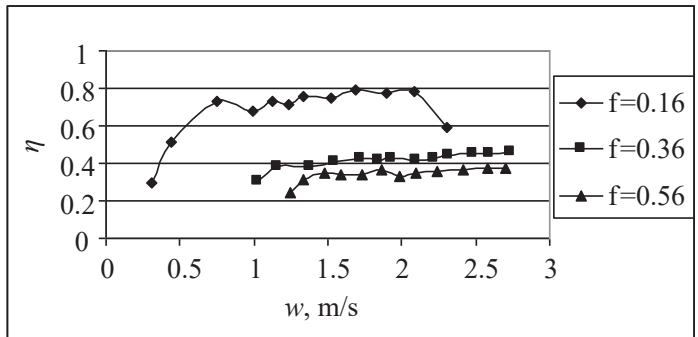

Fig. 1. Dependence of the efficiency of the dual-flow plates on the vapor velocity in the column section (methanol - water (50 mol. \%) system)

Thus, the results of the analysis allow us to conclude that the main parameter that affects the operation of the fail-type trays is their free section.

Fig. 1 shows that the dual-flow plates operate stably in the entire range of existence on the plates of the gas-liquid layer, and at this time only the jet mode of operation of the plates is realized.

For comparison, in Fig. 1 also shows a graph of the dependence of the plate efficiency on the vapor velocity in the column section for a dual-flow plate with a conventional free section $(f=0.16)$. It can be seen that the dual-flow plates with a large cross section operate at higher vapor and liquid loads than plates with a conventional free cross section. At the same time, the efficiency of dual-flow plates with a conventional free section is much higher than the efficiency of plates with a large free section.

In [10], the hydrodynamic characteristics of the dualflow plates with both small and large free cross sections are investigated, although the main attention is paid to the dual-flow plates with a small free cross section. The column diameter is $D=0.057 \mathrm{~m}$.

In [11], the hydraulic characteristics of dual-flow plates with a large free section on columns with a diameter of $D=0.15,0.4$ and $2 \mathrm{~m}$ are investigated. The studies are carried out on a model water-air system. The free section of the plates is $f=0.36$ and 0.56 . The efficiency of the plates is investigated on the system methanol - water 50 mol. \% on a column with a diameter $D=0.3 \mathrm{~m}$. The free section of the plates is $f=0.36$ and 0.56 . A jet operating mode is implemented on the plates.

Thus, the results of the analysis allow to conclude that the main parameter that affects the operation of the dual-flow plates is their free section.

\section{Methods of research}

In this work, dual-flow plates with a large free section $(f=0.36$ and 0.42$)$ are investigated on a laboratory setup described in [10].

The column diameter is $D=0.057 \mathrm{~m}$. The hydraulic resistance of the plate and the height of the gas-liquid layer are measured. The studies are carried out in a wide range of gas and liquid loads on a model air - water system.

\section{Research results}

Fig. 2 shows a graph of the dependence of the hydraulic resistance of a dual-flow flow with a large free section ( $f=0.36)$ depending on the gas velocity in the column section. The studies are carried out on a column with a diameter of $D=0.057 \mathrm{~m}$. For comparison, the graphs of studies of dual-flow plates $(f=0.36)$, carried out on columns with a diameter of $D=0.4 \mathrm{~m}$ and $D=2 \mathrm{~m}$ from [11], are shown.

Fig. 2 shows that all the plates have the same operating range. For irrigation density $L \approx 100 \mathrm{~m}^{3} /\left(\mathrm{m}^{2}\right.$.hour $)$, the plates operate stably at high gas velocities, up to $w=2 \mathrm{~m} / \mathrm{s}$. The plates have close values of the hydraulic resistance, taking into account the absence of a moving gas-liquid layer mode on the plates, since there is no continuous layer of the liquid phase on the dual-flow plates with a large free section [10].

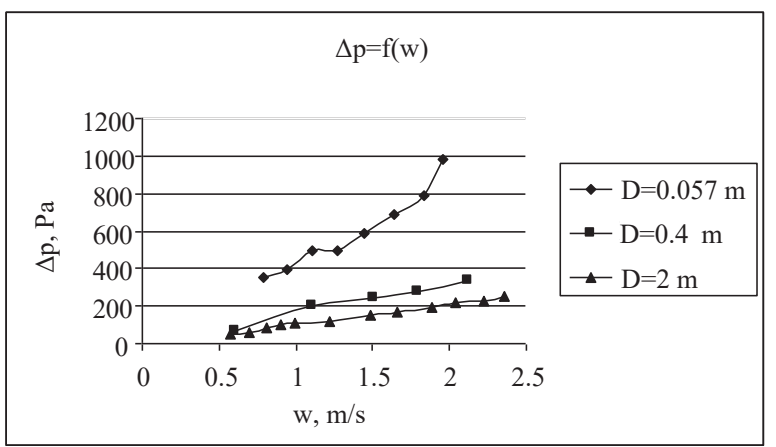

Fig. 2. Dependence of the hydraulic resistance of the dual-flow plates with a large free section $(f=0.36)$ on the vapor velocity in the column section. The irrigation density is $L \approx 100 \mathrm{~m}^{3} /\left(\mathrm{m}^{2}\right.$.hour) 
Fig. 3 shows the graphs of the dependence of the height of the gas-liquid layer on the plate on the gas velocity in the column section for the same conditions as in Fig. 2. The graphs show almost complete coincidence for all dualflow plates with a large free section.

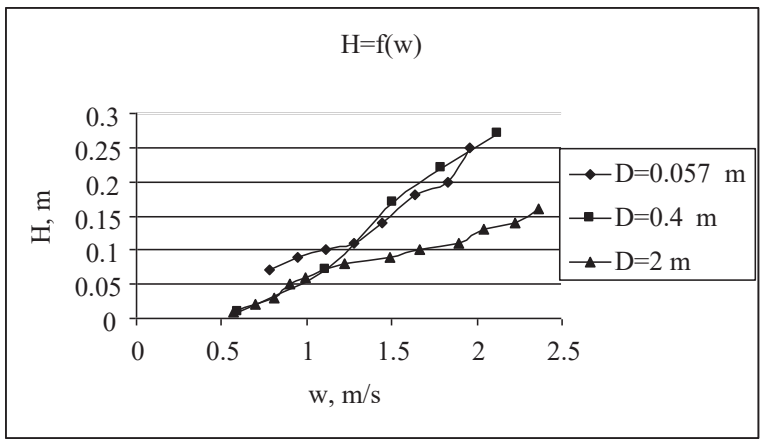

Fig. 3. Dependence of the height of the gas-liquid layer of dual-flow plates with a large free section $(f=0.36)$ on the vapor velocity in the column section. The irrigation density is $L \approx 100 \mathrm{~m}^{3} /\left(\mathrm{m}^{2}\right.$.hour)

Thus, it is possible to say that there is no large-scale transition problem for dual-flow plates with a large free section.

A column with a diameter of $D=0.057 \mathrm{~m}$ can be used to simulate absorption processes if large loads on the liquid phase and low hydraulic resistance of the column apparatus are required.

\section{SWOT analysis of research results}

Strengths. It is found that the dual-flow plates with a large free section operate at high velocities, both in the gas and liquid phases, in comparison with plates with a small free section. This allows them to be installed in columns of smaller diameters. The operation of the dualflow plates with a large free section does not depend on the diameter of the column apparatus. This makes it possible to use a pilot plant with a small column diameter for hydrodynamic modeling of such plates.

Weaknesses. It is shown that under the jet mode of operation, the efficiency of the dual-flow plates with a large free section decreases. And at high gas velocities on such plates, the amount of spray loss increases.

Opportunities. Separators must be installed in the separation space between the plates. They will play the role of an additional zone of phase contact, which will increase the overall efficiency of the contact stage.

In the world, there is little use of the dual-flow plates with a large free section, so they are little studied. However, the study of such plates will be useful for other countries in view of their positive aspects.

Threats. Additional costs will be required for the manufacture of separators and their installation.

\section{Conclusions}

1. The range of operation of dual-flow plates with a large free section is established. The minimum operating speed is $w_{\min }=0.6 \mathrm{~m} / \mathrm{s}$, and the maximum is $w_{\max }=2 \mathrm{~m} / \mathrm{s}$ at an irrigation density $L \approx 100 \mathrm{~m}^{3} /\left(\mathrm{m}^{2}\right.$.hour $)$

2. The study of the dependence of the hydraulic resistance of the dual-flow plates with a large free section on the gas velocity in the column section for the irrigation density $L \approx 100 \mathrm{~m}^{3} /\left(\mathrm{m}^{2}\right.$.hour $)$ is carried out. The gas velocity in the column section reaches $w=2 \mathrm{~m} / \mathrm{s}$.

3. The study of the dependence of the height of the gas-liquid layer of the dual-flow plates with a large free section on the gas velocity in the column section for the irrigation density $L \approx 100 \mathrm{~m}^{3} /\left(\mathrm{m}^{2}\right.$.hour $)$ is carried out. The gas velocity in the column section reaches $w=2 \mathrm{~m} / \mathrm{s}$.

4. It is shown that the dual-flow plates with a large free section can be used for irrigation density up to $L \approx 100 \mathrm{~m}^{3} /\left(\mathrm{m}^{2} \cdot\right.$ hour $)$. In this case, the gas velocity in the column section can be $w=2 \mathrm{~m} / \mathrm{s}$.

\section{References}

1. Syeda, S. R., Afacan, A., Chuang, K. T. (2007). A Fundamental Model for Prediction of Sieve Tray Efficiency. Chemical Engineering Research and Design, 85 (2), 269-277. doi: http:// doi.org/10.1205/cherd06111

2. Bubble Tray Design Manual (1958). New York: American Institute of Chemical Engineers, 94.

3. Chan, H., Fair, J. R. (1984). Prediction of point efficiencies on sieve trays. 1. Binary systems. Industrial $\mathcal{E}$ Engineering Chemistry Process Design and Development, 23 (4), 814-819. doi: http://doi.org/10.1021/i200027a032

4. Chen, G. X., Chuang, K. T. (1993). Prediction of point efficiency for sieve trays in distillation. Industrial $\mathcal{E}$ Engineering Chemistry Research, 32 (4), 701-708. doi: http://doi.org/10.1021/ie00016a017

5. Diener, D. A. (1967). Calculation of Effect of Vapor Mixing on Tray Efficiency. Industrial $\mathcal{E}$ Engineering Chemistry Process Design and Development, 6 (4), 499-503. doi: http://doi.org/ 10.1021/i260024a018

6. Gerster, J. A., Hill, A. B., Hochgraf, N. N., Robinson, D. G. (1958). Tray Efficiencies In Distillation Columns American Institute of Chemical Engineers (AIChE). Final report from the University of Delaware. New York, 118.

7. Bennett, D. L., Agrawal, R., Cook, P. J. (1983). New pressure drop correlation for sieve tray distillation columns. AIChE Journal, 29 (3), 434-442. doi: http://doi.org/10.1002/aic.690290313

8. Mukhlenov, I. P., Tarat, E. Ia., Tubolkin, A. F., Tumarkina, E. S. (1977). Pennii rezhim i pennye apparaty. Leningrad: Khimiia, 304.

9. Taranenko, G. V. (2019). Gas content on dual-flow plate in a combined contact device. ScienceRice, 1 (54), 19-25. doi: http:// doi.org/10.15587/2313-8416.2019.155858

10. Taranenko, G. V. (2013). Gidravlicheskie i massoobmennye kharakteristiki tarelok provalnogo tipa s razlichnym diametrom otverstii. Lugansk: VNU im. V. Dalia, 174.

11. Taranenko, G. V. (1984). Gidrodinamika i massoperedacha kombinirovannogo kontaktnogo ustroistva. GIAP, 298.

Taranenko Gennadiy, PhD, Associate Professor, Department of Mechanical Engineering and Equipment Industry, Volodymyr Dahl East Ukrainian National University, Severodonetsk, Ukraine, ORCID: http:// orcid.org/0000-0001-8240-6773,e-mail: gtaranenko@ukr.net 